\title{
A novel two-layer, intradural and extradural patch graft approach to treating dural defects and tears: illustrative case
}

\author{
Daniella Lazarus, BS, ${ }^{1}$ Charlotte Hawks, MS, ${ }^{1}$ Namrita Kumar, ${ }^{1}$ Tara McCaffrey, BA, ${ }^{1}$ and Arthur L. Jenkins III, MD ${ }^{1,2,3}$ \\ ${ }^{1}$ Jenkins NeuroSpine, New York, New York; and Departments of ${ }^{2}$ Orthopedics and ${ }^{3}$ Neurosurgery, Icahn School of Medicine, The Mount Sinai Hospital, New York, New York
}

BACKGROUND Dural tears must be quickly addressed to avoid the development of positional headaches and pseudomeningoceles, among other complications. However, sizeable areas of friable or absent dura create unique challenges when attempting to achieve a watertight seal. We have developed a two-layer subdural and epidural fibrous patch technique to treat expansive or challenging dural tears as a result of our experience treating spinal fluid leaks.

OBSERVATIONS The authors present the treatment of a large necrotic $(5 \times 1.5 \mathrm{~cm})$ dural defect refractory to initial attempts at standard primary repair with dural patch grafting and requiring a revision with a dual-layer patch to manage persistent cerebrospinal fluid leakage.

LESSONS The use of a two-layer (subdural and epidural) patch is both a safe and effective dural repair technique for creating a watertight seal in challenging large areas in which the dura may be damaged, scarred, or absent. We also propose that this technique may be able to be used for smaller challenging tears, as well as potentially for repairs of large blood vessels or other fluid-filled structures in the body.

https://thejns.org/doi/abs/10.3171/CASE21639

KEYWORDS dura; dural repair; graft; spinal fluid leak

Cerebrospinal fluid (CSF) leaks can lead to long-term, debilitating consequences if inappropriately treated. Those suffering from a CSF leak typically present with positional headaches and often nausea, vomiting, dizziness, photophobia, phonophobia, neck pain, and stiffness. ${ }^{1,2}$ Possible consequences include pseudomeningoceles, secondary Chiari malformations, arachnoiditis, infections, and nerve dysfunction. ${ }^{1,3}$

CSF leaks, recognized intraoperatively or through imaging, can be managed conservatively or surgically. ${ }^{4}$ Conservative management includes bed rest, an epidural blood patch, and/or fibrin glue. ${ }^{1,5}$ Those patients for whom conservative management fails may require a neurosurgeon to either suture the tear closed, use a dural sealant such as fibrin glue, place a fat or muscle graft, insert a patch, or use a combination of techniques to achieve a watertight closure. ${ }^{6}$ The specific repair depends on the length, location, and cause of the leak.

Patch materials, made of nonporous fibrous material that can withstand sutures and other graft materials, have been proposed and used to ensure a watertight dural repair. Materials such as AlloDerm (Allergan; suturable Duragen [Integra]; and Gore-Tex [Gore]) have been found to be effective as a single-layer patch. ${ }^{7}$ However, in some cases, the edges of the native dura may be so friable that suturing or clipping to the dura results in incomplete seals, tears, or leaks through the suture holes. ${ }^{4,8}$ Additionally, those with a history of radiotherapy to or near the spine may have compromised dural blood supply, extensive scarring or adhesions, and/or tissue hypoxia, thereby weakening the dura mater, increasing the likelihood of CSF leaks, and requiring a more intensive dural closure. ${ }^{4,8}$ Thus, this study presents a novel approach to treating a radiation-induced dural injury and CSF leak as well as expansive dural tears by the use of a two-layer intradural and extradural patch graft.

\section{Illustrative Case}

A 61-year-old female patient presented with a history of colon cancer with metastases to the T12 rib. Upon diagnosis, she underwent an intralesional resection and radiotherapy, which resulted in significant dosage to the vertebral body and thecal sac. After the tumor recurred with invasion of the vertebral body, an en bloc resection was attempted. 
The tumor was larger than anticipated and could not be removed completely, resulting in a tumor recurrence to the right chest and flank area. Her postoperative recovery was complicated by several falls, fractures of the vertebral bodies and pelvis, and loosening of the screws.

Throughout the year, she required several revisions of the hardware construct and wound as well as placement of a myocutaneous flap to manage hardware prominence. She also received repeat radiation to the area of recurrence, which reduced her pain but resulted in necrosis of the skin and muscle flap placed during her last procedure. She ultimately presented with a gaping wound with fluid draining from it and exposed hardware, spine, and dura and was admitted to undergo a three-part procedure to repair the wound and stabilize the spine.

During the first operation, clear fluid was seen seeping from a small hole in the lateral aspect of the spinal dural sac around right L2. 6-0 Gore-Tex sutures and a muscle patch were used to seal this durotomy, however, the repair was unsuccessful.

One week later, the patient returned to have the dura surgically reconstructed. CSF was seen leaking from multiple locations with necrotic or no dura over a large area $(1.5 \times 5 \mathrm{~cm})$ on the right side of the dura tube, stretching from L1 to L3. The necrotic edges were resected back to more solid dural tissue (Fig. 1). We fashioned an AlloDerm dual-layer patch that was approximately $2.5 \mathrm{~cm}$ wide by 7 $\mathrm{cm}$ long on the inner layer and $1.5 \mathrm{~cm}$ wide by $5 \mathrm{~cm}$ long on the outer layer with the outer layer mimicking the teardrop shape of the dural defect (Fig. 2). These two layers were sutured together in five places to maintain alignment. The inner layer was inserted so that the excess flaps of AlloDerm were in the subdural space while the outer layer was aligned with the dural defect. We sutured the outer layer to the edges of the dura with 6-0 Gore-Tex sutures (Fig. 3).

Upon performing a Valsalva maneuver, no CSF leak was noted. A layer of DuraSeal Exact (Integra) spinal sealant was sprayed on the surface of the patch covering the entire repair. No further CSF leak was seen despite repeat Valsalva maneuvers.

Eight days after the procedure, she returned to the operating room for debridement of the deep wound of the right flank. Multiple Valsalva maneuvers were performed, and some of the DuraSeal Exact was removed without any apparent leakage. Postoperatively, the patient did not require any additional dural intervention nor did she suffer from CSF leak symptoms.

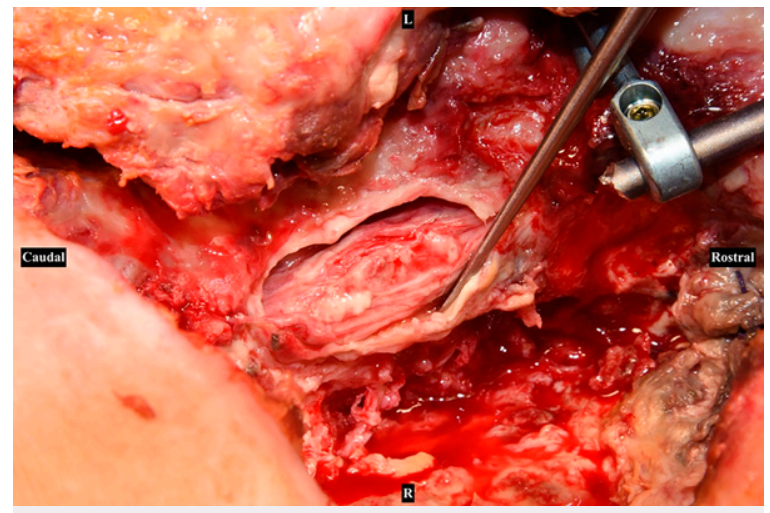

FIG. 1. The subject's open durotomy at T11 to L1. The necrotic and friable dura had already been resected. The T11 and T12 nerve roots had been previously sacrificed. Previous implanted hardware had been cut to open the field of view.

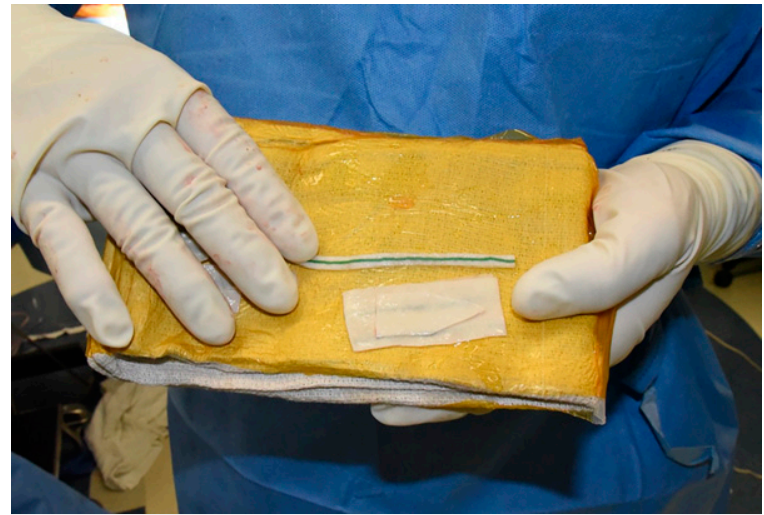

FIG. 2. The implanted AlloDerm dual-layer patch. The larger layer was inserted into the subdural space while the smaller, teardrop-shaped layer was secured along the outer dural wall in exact alignment with the defect, while the two layers were secured to each other at five points in-between.

\section{Discussion}

Dural tear repairs are challenging, however, those with friable dura create a particular issue. ${ }^{8} \mathrm{~A}$ watertight closure is required to avoid debilitating headaches and other complications. ${ }^{6}$ For patients with especially friable or absent dura, closure with the use of sutures or glue may be ineffective. Therefore, other methods must be considered.

A previous case report describes a double-layered duraplasty technique with the use of lyophilized hydrogel sealant. ${ }^{6}$ In this case, intradural and extradural Lyoplant (Aesculap) patches along with DuraSeal were used to treat two large ventral dural tears. ${ }^{8}$ These tears were initially treated with a fat graft and fibrin glue.

However, given the complex tear, the repairs did not provide a long-term watertight closure of the dura. This study demonstrates the use of intradural and extradural patches for ventral, inaccessible CSF leaks, whereas our case study promotes the use of a fused dual-layer technique for large areas of damaged or absent dura.

Because the patch consists of extra material, the dural patch can act as an expansive duroplasty, rather than constricting the intradural space by the suture line of a traditional linear closure.

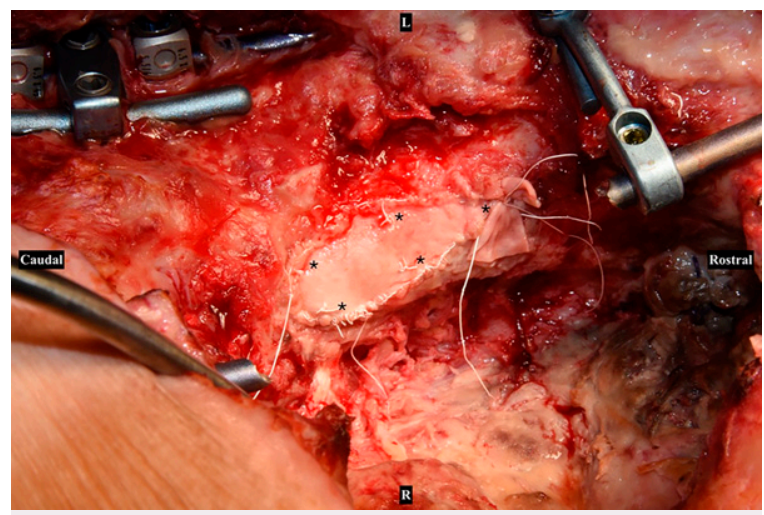

FIG. 3. The sealed durotomy at T11 to L1. The dual-layer patch had been inserted into the durotomy and sutured along the edges of the dural defect as well as fastened at five different points indicated by an asterisk. 


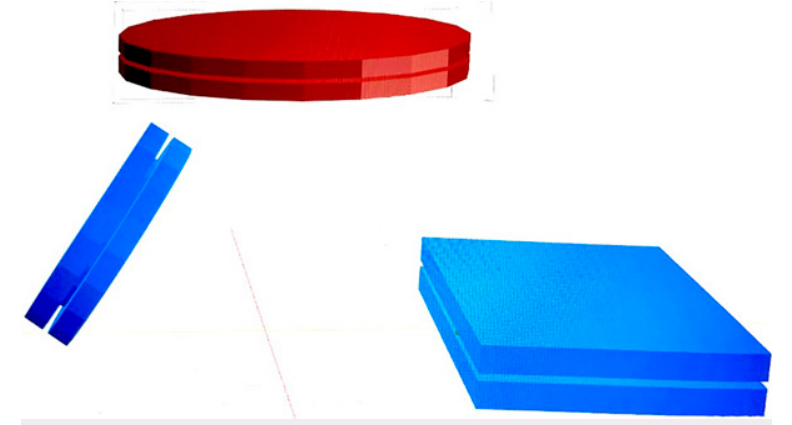

FIG. 4. Three versions of the potential commercial versions of this constructed dural or vascular patch material, consisting of a pair of layers of waterproof, flexible material with a thin layer between them that allows the other two layers to be tightly bound and give extra strength to the overall construct. One square, one oval, and one round construct, each with the two outer layers the same size and shape.

This has the potential to reduce the risk of making the area stenotic (and therefore less likely to cause cord compression) by the added layer of dura in the subdural space. Because the material is only approximately $1 \mathrm{~mm}$ thick, this size implant is generally well tolerated in all but the most congenitally tight canals. Care must be taken not to allow for additional epidural compression to ensue.

Postoperative imaging could be done to look for adequate space for the spinal cord or nerve roots, but given the loss of CSF in these cases, it might not be obvious with intraoperative imaging whether the CSF spaces are preserved or not until the subarachnoid space is fully repressurized.

\section{Observations}

Autologous grafts present challenges, despite being commonly used to treat intraoperative CSF leaks. They may lead to the formation of scar tissue, be inadequate in creating a watertight seal, or may not be available at the time of repair. ${ }^{6}$ For a small dural defect, there should be a sufficient amount of fat or muscle to seal the tear.

However, for a large tear, multiple small tears, or areas in which the dura is absent, fat or muscle grafts of this size or quantity are usually not available. ${ }^{9}$ Extensive grafts increase the likelihood of experiencing mass effect, thereby pushing on or displacing nearby structures such as the spinal cord. ${ }^{6}$ In our case, the patient did remarkably better with this technique in comparison to her prior CSF leak repair.

\section{Lessons}

We therefore propose this novel two-layer intradural and extradural patch technique utilizing a nonporous tissue matrix (such as AlloDerm or suturable Duragen) in order to achieve a watertight closure when one or more large areas of the dura are friable, thin, or absent. By having the inner layer larger than the defect, the two-layer patch technique creates a nonporous overlay of the repair on the side of the higher pressure state. This technique can also be applied to expand the dura for the prevention of intradural adhesions and for the repair of previously damaged dura, or for repair of large blood vessels with a defect in the wall.

The bilayer technique, either with two layers sutured together, or as a bilayer constructed or fused together (Fig. 4, patent pending), creates a watertight seal by having a layer over the defect and a layer inside the lumen pushed into place by the higher-pressure environment. These materials are typically not thick enough to cause significant compression on the neural elements.

The outer layer can be cut and contoured to the actual defect (as in this case) or left larger to allow for suturing to more robust dura away from the edge. In either case, closure of the outer layer keeps the inner layer in place while doubling the thickness of the repair.

Much smaller versions of fused bilayer patches could be used for primary closure of smaller holes or leaks without sutures. An inner layer of nonporous material would be inserted and unfold while the center (essentially a thin middle layer) fills most of the defect and prevents separation of the layers. The outer layer could be sealed with dural glue or sutures. This technique could save time and improve the chances of repairs. Overall, this bilayer technique may improve dural repair for all but the simplest defects.

\section{References}

1. Jankowitz BT, Atteberry DS, Gerszten PC, et al. Effect of fibrin glue on the prevention of persistent cerebral spinal fluid leakage after incidental durotomy during lumbar spinal surgery. Eur Spine J. 2009;18(8):1169-1174.

2. Mokri B. Spontaneous intracranial hypotension. Curr Pain Headache Rep. 2001;5(3):284-291.

3. Atkinson JL, Weinshenker BG, Miller GM, Piepgras DG, Mokri B. Acquired Chiari I malformation secondary to spontaneous spinal cerebrospinal fluid leakage and chronic intracranial hypotension syndrome in seven cases. J Neurosurg. 1998;88(2):237-242.

4. Iannella G, Manno A, Pasqualitto E, et al. Massive cerebrospinal fluid leak of the temporal bone. Case Rep Otolaryngol. 2016; 2016:7521798.

5. Lin W, Geiderman J. Myth: fluids, bed rest, and caffeine are effective in preventing and treating patients with post-lumbar puncture headache. West J Med. 2002;176(1):69-70.

6. Lee DH, Kim KT, Park JI, Park KS, Cho DC, Sung JK. Repair of inaccessible ventral dural defect in thoracic spine: double layered duraplasty. Korean J Spine. 2016;13(2):87-90.

7. Danish SF, Samdani A, Hanna A, Storm P, Sutton L. Experience with acellular human dura and bovine collagen matrix for duraplasty after posterior fossa decompression for Chiari malformations. $J$ Neurosurg. 2006;104(1)(suppl):16-20.

8. Yokogawa N, Murakami $\mathrm{H}$, Demura $\mathrm{S}$, et al. Effects of radiation on spinal dura mater and surrounding tissue in mice. PLoS One. 2015;10(7):e0133806.

9. Black P. Cerebrospinal fluid leaks following spinal surgery: use of fat grafts for prevention and repair. Technical note. J Neurosurg. 2002;96(2)(suppl):250-252.

\section{Disclosures}

Dr. Jenkins reported a patent for Surgical Implant for Repairing a Defect in Spinal Dura Mater is pending. No other disclosures were reported.

\section{Author Contributions}

Conception and design: Jenkins, Lazarus, Hawks. Acquisition of data: Jenkins, Lazarus, Kumar. Analysis and interpretation of data: Jenkins, Lazarus, Hawks. Drafting the article: all authors. Critically revising the article: all authors. Reviewed submitted version of manuscript: all authors. Approved the final version of the manuscript on behalf of all authors: Jenkins. Administrative/technical/material support: Jenkins, Lazarus, McCaffrey. Study supervision: Jenkins.

\section{Correspondence}

Arthur L. Jenkins III: Jenkins NeuroSpine, New York, NY. alj@ arthurjenkinsmd.com. 